University of Wollongong

Research Online

Faculty of Engineering and Information

Faculty of Engineering and Information

Sciences - Papers: Part A

Sciences

$1-1-2014$

Thermodynamic model of coherent island formation on vicinal substrate

Xu Zhang

Zhengzhou University

Yanguang Yu

University of Wollongong, yanguang@uow.edu.au

Xiao-Hong Sun

Zhengzhou University

Xiaomin Ren

Beijing University of Posts and Telecommunications

Follow this and additional works at: https://ro.uow.edu.au/eispapers

Part of the Engineering Commons, and the Science and Technology Studies Commons

Research Online is the open access institutional repository for the University of Wollongong. For further information contact the UOW Library: research-pubs@uow.edu.au 


\title{
Thermodynamic model of coherent island formation on vicinal substrate
}

\author{
Abstract \\ A thermodynamic model has been proposed to address the formation of coherent island on the vicinal \\ substrate. The morphological transition from square based island to elongated based one with various \\ substrate misorientations is described. The initial stage of nucleation and growth process of islands in \\ Stranski-Krastanow system is studied by taking into account the elastic deformations and the change of \\ energy in the case of two-dimensional growth mode. The theoretical analysis shows the minimum \\ nucleation barrier of island is on the decrease with increment of substrate misorientation, which means \\ the nucleation of island on vicinal substrate is more favorable than that on flat substrate. By using the \\ fitting data of experimental results done by Persichetti et al., [Phys. Rev. Lett. 104, 036104 (2010) and \\ Phys. Rev. B 82, 121309(R) (2010)], we provide a meaningful explanation of the experimental \\ observations. \\ Disciplines \\ Engineering | Science and Technology Studies

\section{Publication Details} \\ $X$. Zhang, Y. Yu, X. Sun \& X. Ren, "Thermodynamic model of coherent island formation on vicinal \\ substrate," Journal of Applied Physics, vol. 115, (16) pp. 163508-1 - 163508-5, 2014.
}

This journal article is available at Research Online: https://ro.uow.edu.au/eispapers/3332 


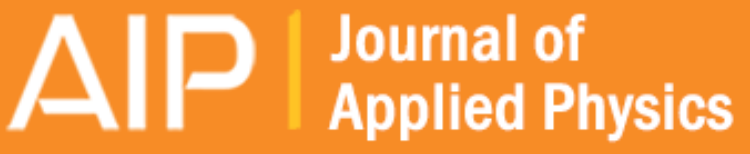

\section{Thermodynamic model of coherent island formation on vicinal substrate}

Xu Zhang, Yanguang Yu, Xiao-Hong Sun, and Xiaomin Ren

Citation: Journal of Applied Physics 115, 163508 (2014); doi: 10.1063/1.4873458

View online: http://dx.doi.org/10.1063/1.4873458

View Table of Contents: http://scitation.aip.org/content/aip/journal/jap/115/16?ver=pdfcov

Published by the AIP Publishing

\section{Articles you may be interested in}

Solution-growth kinetics and thermodynamics of nanoporous self-assembled molecular monolayers

J. Chem. Phys. 134, 124702 (2011); 10.1063/1.3569132

Implantation conditions for diamond nanocrystal formation in amorphous silica

J. Appl. Phys. 104, 034315 (2008); 10.1063/1.2968204

Elastoplastic phase field model for microstructure evolution

Appl. Phys. Lett. 87, 221910 (2005); 10.1063/1.2138358

Phase transition, thermodynamic properties, and microstructure of $\mathrm{Fe}-\mathrm{Pt}$ based alloys

J. Appl. Phys. 93, 7586 (2003); 10.1063/1.1538175

Relaxation model of coherent island formation in heteroepitaxial thin films

J. Appl. Phys. 88, 4586 (2000); 10.1063/1.1311305

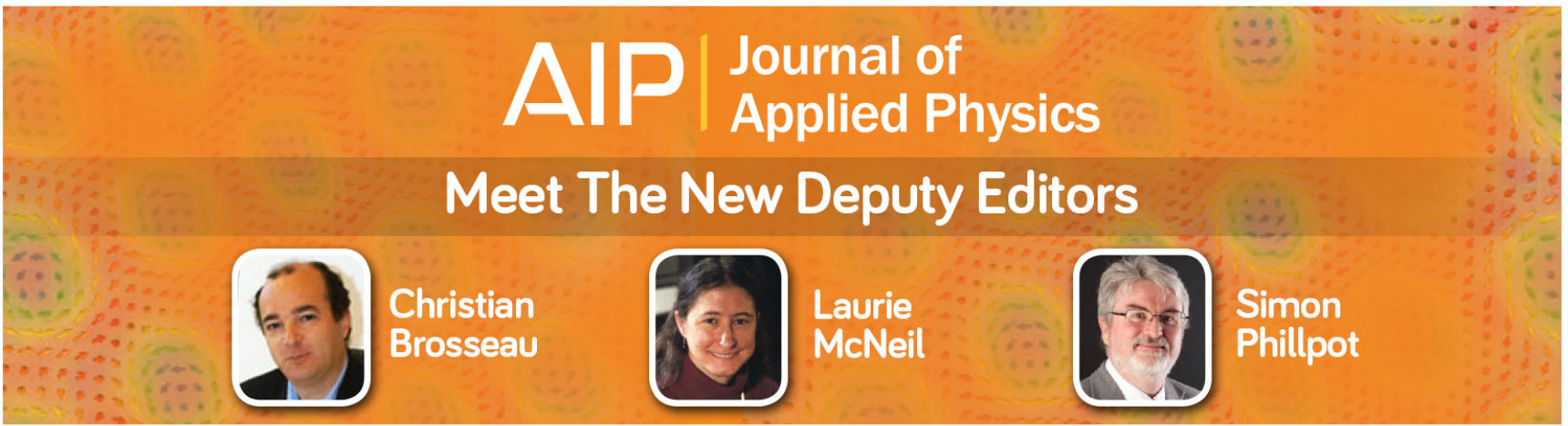




\title{
Thermodynamic model of coherent island formation on vicinal substrate
}

\author{
Xu Zhang, ${ }^{1, a)}$ Yanguang Yu, ${ }^{2}$ Xiao-Hong Sun, ${ }^{1}$ and Xiaomin Ren $^{3}$ \\ ${ }^{1}$ Henan Key Laboratory of Laser and Opto-electric Information Technology, School of Information \\ Engineering, Zhengzhou University, Zhengzhou 450052, China \\ ${ }^{2}$ School of Electrical, Computer and Telecommunications Engineering, University of Wollongong, Northfields \\ Ave, Wollongong, New South Wales 2522, Australia \\ ${ }^{3}$ State Key Laboratory of Information Photonics and Optical Communications, Beijing University of Posts and \\ Telecommunications, Beijing 100876, China
}

(Received 8 December 2013; accepted 15 April 2014; published online 24 April 2014)

\begin{abstract}
A thermodynamic model has been proposed to address the formation of coherent island on the vicinal substrate. The morphological transition from square based island to elongated based one with various substrate misorientations is described. The initial stage of nucleation and growth process of islands in Stranski-Krastanow system is studied by taking into account the elastic deformations and the change of energy in the case of two-dimensional growth mode. The theoretical analysis shows the minimum nucleation barrier of island is on the decrease with increment of substrate misorientation, which means the nucleation of island on vicinal substrate is more favorable than that on flat substrate. By using the fitting data of experimental results done by Persichetti et al., [Phys. Rev. Lett. 104, 036104 (2010) and Phys. Rev. B 82, 121309(R) (2010)], we provide a meaningful explanation of the experimental observations. (C) 2014 AIP Publishing LLC. [http://dx.doi.org/10.1063/1.4873458]
\end{abstract}

\section{INTRODUCTION}

The spontaneous formation of coherent islands in heteroepitaxial systems is of high technological relevance as it is a major method to produce dense arrays of nanoscale quantum dots (QDs). ${ }^{1,2}$ Self-assembly of QDs have attracted much interest due to their original physical properties and potential device applications. ${ }^{2-4}$ Although the main mechanisms of heteroepitaxial growth of QDs on flat substrate have been elucidated, ${ }^{5,6}$ the vicinal systems exhibit many different features in the experimental observations which are of vital importance to be investigated theoretically. Controlling the size, shape, and a predictable spatial ordering of QDs on the vicinal substrate remains significant challenges, in part because of a limited understanding of the mechanisms of strain-induced transition from wetting layer to faceted QDs. On the other hand, miscut offers another way of control over the shape and properties of selfassembled QDs. In order to control the morphology of growing islands, it is necessary to find out which factors play an important role in the thermodynamic process. It is an urgent issue to be solved, because in their practical use in optoelectronics, the optical properties of QDs are largely determined by the island shape and size distribution.

The thermodynamic model of coherent island on the flat substrate has already been established by Dubrovskii et al. and Osipov et al. ${ }^{5,6}$ It includes the surface energy, the elastic strain energy, and the attractive energy of the wetting layer. Though their proposed model has successfully explained the nucleation barrier and size distribution of island; however, it fails to take into account the thickness-dependent surface energy of wetting layer, which can not be regarded as constant in practical deposition process. Hence, a further

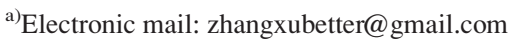

development of relevant theoretical model could help in an understanding of the overall vicinal system performance.

Recently, Persichetti et al..$^{7,8}$ have obtained a lot of experimental results of $\mathrm{Ge}$ islands on vicinal $\mathrm{Si}(001)$ substrate. By fitting his finite element calculations of elastic energy density of Ge islands and the shape transition of Ge islands as a function the miscut angle, we propose a quantitative thermodynamic model of coherent island formation on vicinal substrate. This model takes into account both the thickness-dependent surface energy of wetting layer and the attraction energy of wetting layer to the island. Our theoretical expectation about the nucleation barrier is consistent with experimental observations, which implies that the proposed thermodynamic theory could be applicable to the selfassembled islands on the strained vicinal substrate.

\section{THERMODYNAMIC THEORY}

During the layer-by-layer heteroepitaxial deposition process, the lattice mismatch induced strain stored in the epitaxial wetting layer cannot be maintained and needs to be released as the wetting layer continues to grow. ${ }^{9}$ Thus, in order to effectively release the strain, the nucleation process of $3 \mathrm{D}$ islands will take place on the wetting layer when the thickness of it exceeds a critical value. Now, the relaxation of elastic stress occurs. From the thermodynamic point of view, the change from layer-by-layer growth to the formation of 3D islands is due to the lower energetic value of system. In the following, we develop an analytical description of the nucleation and growth process of islands on the vicinal substrate. For the case of 3D coherent island growing on the vicinal substrate as shown in Fig. 1, the free energy formation in Stranski-Krastanow (SK) growth mode can be presented in the form

$$
\Delta E(V, \theta)=\Delta E_{\text {elastic }}+\Delta E_{\text {surf }}+\Delta E_{\text {wet }} .
$$




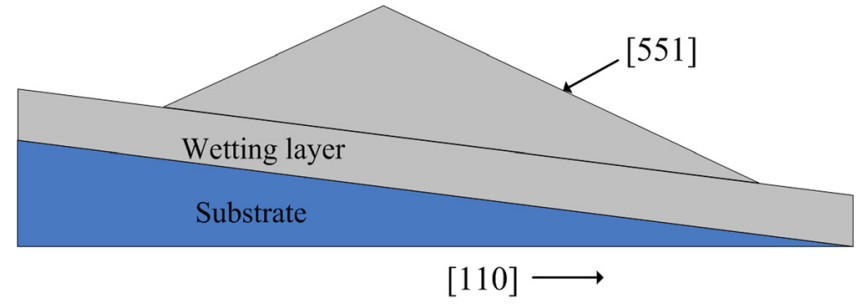

FIG. 1. Schematic illustration an island on the vicinal substrate.

Here, $\Delta E_{\text {elastic }}$ is the difference in elastic energy of the island and the wetting layer, $\Delta E_{\text {surf }}$ is the difference in surface energy of island and of wetting layer surface covered by this island, and $\Delta E_{\text {wet }}$ is the change of energy in the case of layerby-layer growth mode on a strained substrate, which always takes place in SK growth mode. The base of the island is represented according to the experimental results, the ratio of the longest island side to the shortest one $f=L_{m} / L_{M}$ as a function of miscut angle $\theta$ is fitted as $f=-45 \theta / 2 \pi+1$, $(0<\theta<\pi / 30),{ }^{15}$ so $L_{m}=L_{M}(2 \pi-45 \theta) / 2 \pi$.

In our theoretical work, we investigated the system of Ge islands on $\operatorname{Si}(001)$ substrate. Previous works show that the Ge islands always demonstrate $\{105\}$ facets on the flat $\mathrm{Si}(001)$ substrate. According the experimental result, $\{105\}$ facets are also energetically favored on misoriented $\mathrm{Si}(001)$ substrate. ${ }^{10}$ In Ge island, the [551] intersection line of adjacent $\{105\}$ facets forms a $8.05^{\circ}$ angle with the (001) plane. To allow $\{105\}$ faceting, this angle must never change, producing the observed elongation toward the miscut direction, as schematically illustrated in Fig. 2. When the miscut angle is larger than $8.05^{\circ}$, a pyramidal shape can no longer form, since the [551] intersection line runs parallel to the substrate orientation, and the islands morphology rearranges itself into ripples. ${ }^{8}$ This kind of special case is not included in our paper, and will be discussed in future work.

The first term in Eq. (1) is given by

$$
\Delta E_{\text {elastic }}=\left(e_{\text {island }}^{e l}-W_{0}\right) V
$$

where $e_{i s l a n d}^{e l}$ is the elastic energy density of the island, $V$ is the volume of quantum dot, and $W_{0}=E \varepsilon_{0}^{2} /(1-\nu)$ is the elastic strain energy density in a flat uniformly strained film. $E$ is the Young's modulus, $\varepsilon_{0}$ is the misfit parameter, and $v$ is the Poisson ratio. The fit to the finite element calculation of the elastic energy density of islands on various miscut substrate takes a form of $e_{i s l a n d}^{e l}=W_{0}-a(1+\nu) W_{0} \tan \vartheta,{ }^{11}$ where $\vartheta$ is the tilt angle of the island side surface on the flat substrate,

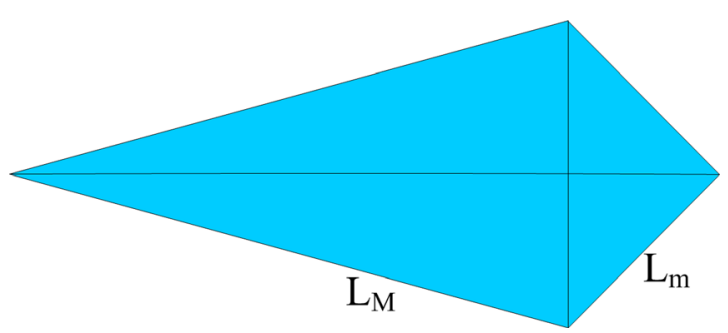

FIG. 2. Illustration of the base of the elongated pyramid. $L_{m}$ and $L_{M}$ are the shortest and longest island side, respectively. for $\mathrm{Ge} / \mathrm{Si}(00)$ system, the $\{105\}$ side facets on the flat $\mathrm{Si}(001)$ is energetically favorable, in this case, $\vartheta=11.3^{\circ}$. The coefficient $a=0.954$ which is found by fitting $e_{i s l a n d}^{e l}$ with the numerical value of FEM data ${ }^{7}$ under the condition of $\vartheta=11.3^{\circ}$, is a little smaller than Shchukin's result of $a=0.9922 .{ }^{11}$ The equivalent value of $\vartheta$ of the elongated QDs on the vicinal substrate obtained based on the FEM results equal $11.3^{\circ}, 11.28^{\circ}$, $11.2^{\circ}, 11^{\circ}, 10.68^{\circ}, 10.25^{\circ}, 9.7^{\circ}$ for the miscut angles of $0^{\circ}, 1^{\circ}$, $2^{\circ}, 3^{\circ}, 4^{\circ}, 5^{\circ}, 6^{\circ}$, respectively (Fig. 3).

Surface energy contribution to $\Delta E(V, \theta)$ is determined by

$$
\Delta E_{\text {surf }}=\gamma_{f} A_{2}(\theta) V^{2 / 3}-\gamma\left(\theta, H_{W L}\right) A_{1}(\theta) V^{2 / 3},
$$

where $A_{1}(\theta)$ and $A_{2}(\theta)$ are the shape factors, which are determined by the shape of the island. $H_{W L}$ is the wetting layer thickness, $\gamma_{f}$ is the surface energy density of the island side facet, $\gamma(\theta, H)=\gamma_{0}(H) \cos \theta+\beta \sin \theta$ is the surface energy density of WL on the miscut substrate, and $\beta=$ $\left[\gamma_{0}(H)-\gamma_{0}(H) \cos \varphi\right] \csc \varphi$ is the step formation energy per unit height. ${ }^{12}$ A lower bound on $\beta$ is the value for a facet of neighboring orientation $\varphi$ (here is $11.3^{\circ}$ ). The thicknessdependent surface energy density of WL on the flat substrate can be expressed as the function of thickness $H$ monolayers (ML, number of atomic layers), $\gamma_{0}(H)=\gamma_{s u b}^{\infty}+\left(\gamma_{W L}^{\infty}\right.$ $\left.-\gamma_{s u b}^{\infty}\right)(1-\exp (-H / \delta)){ }^{13}$ where $\gamma_{s u b}^{\infty}$ and $\gamma_{W L}^{\infty}$ are the surface energy density of substrate and WL with infinite thickness and $\delta$ is a dimensionless parameter that depends on the interaction between layers.

The energy change in the case of layer-by-layer growth mode in Eq. (1) is represented as ${ }^{18}$

$$
\Delta E_{w e t}=\frac{1}{n} \times\left[\gamma\left(\theta, H_{W L}\right)-\gamma\left(\theta, H_{0}\right)\right]
$$

where $n$ is the density of the quantum dots (the number of quantum dots per unit area), $h_{0}$ is the monolayer thickness, $H_{0}=H_{W L}+n V / h_{0}$ represents imaginary thickness of wetting layer where the further deposited material has the same

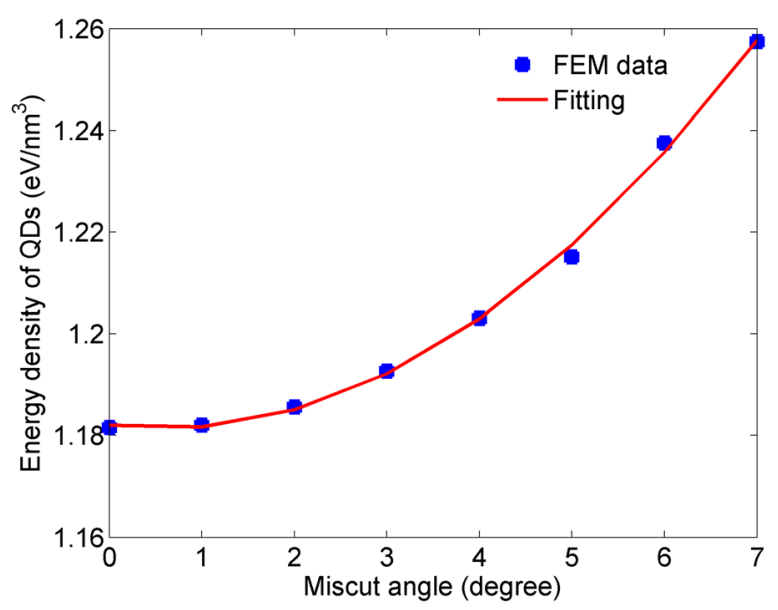

FIG. 3. The elastic energy density of QDs as a function of miscut angle of the substrate, $e_{i s l a n d}^{e l}=W_{0}-a(1+\nu) W_{0} \tan \vartheta$. The equivalent value of $\vartheta$ of the elongated QDs obtained by fitting with the FEM results equals $11.3^{\circ}$, $11.28^{\circ}, 11.2^{\circ}, 11^{\circ}, 10.68^{\circ}, 10.25^{\circ}, 9.7^{\circ}$ for the miscut angles of $0^{\circ}, 1^{\circ}, 2^{\circ}$, $3^{\circ}, 4^{\circ}, 5^{\circ}, 6^{\circ}$, respectively. 
volume as that of QD. The other parameters take the same meaning mentioned above.

Combining Eqs. (2)-(4) in Eq. (1), the final formula of free energy of stress-driven coherent island on the vicinal substrate is written down as

$$
\begin{aligned}
\Delta E= & \gamma_{f} A_{2}(\theta) V^{2 / 3}-\gamma\left(\theta, H_{W L}\right) A_{1}(\theta) V^{2 / 3} \\
& -a(1+\nu) W_{0} V \tan \vartheta+\frac{1}{n} \times\left[\gamma\left(\theta, H_{W L}\right)-\gamma\left(\theta, H_{0}\right)\right] .
\end{aligned}
$$

From the above definition, $\gamma\left(\theta, H_{W L}\right)-\gamma\left(\theta, H_{0}\right)=K(\theta)$ $\exp \left(-H_{W L}\right)\left(\gamma_{\text {sub }}^{\infty}-\gamma_{W L}^{\infty}\right)\left(1-\exp \left(-n V / h_{0}\right)\right)$, in order to obtain of analytical expression of the nucleation barrier, we must simplify the calculation for the surface energy density of wetting layer. Because the volume of island is very small during the deposition process, $n V / h_{0} \ll 1$ and $\left(1-\exp \left(-n V / h_{0}\right)\right)$ can be considered to approximately equal $n V / h_{0}$. In this way, Eq. (5) is expressed as

$$
\begin{aligned}
\Delta E= & \gamma_{f} A_{2}(\theta) V^{2 / 3}-K(\theta) \\
& \times\left[\gamma_{W L}^{\infty}-\left(\gamma_{W L}^{\infty}-\gamma_{\text {sub }}^{\infty}\right) \exp \left(-H_{W L}\right)\right] A_{1}(\theta) V^{2 / 3} \\
& -a(1+\nu) W_{0} V \tan \vartheta+K(\theta) \exp \left(-H_{W L}\right)\left(\gamma_{\text {sub }}^{\infty}-\gamma_{W L}^{\infty}\right) \frac{V}{h_{0}},
\end{aligned}
$$

where $K(\theta)=\cos (\theta)+\csc (\varphi) \sin (\theta)-\csc (\varphi) \sin (\theta) \cos (\varphi)$. It shows the stability of mechanism of competition between the surface energy and relaxation energy during the growth process. The gain in elastic energy is the driving force of QDs growth. As the increase of QD volume, the surface energy increases as well, which prevents QD from growing up. In the initial stage of the nucleation process, the surface energy of QD's side facet plays an important role to prevent QD formation due to high ratio of surface to volume. However, this situation is totally opposite when the volume of QD exceeds a critical value. Hence, the growth of QDs needs the expense the wetting layer, which results in the decrease of the wetting layer thickness.

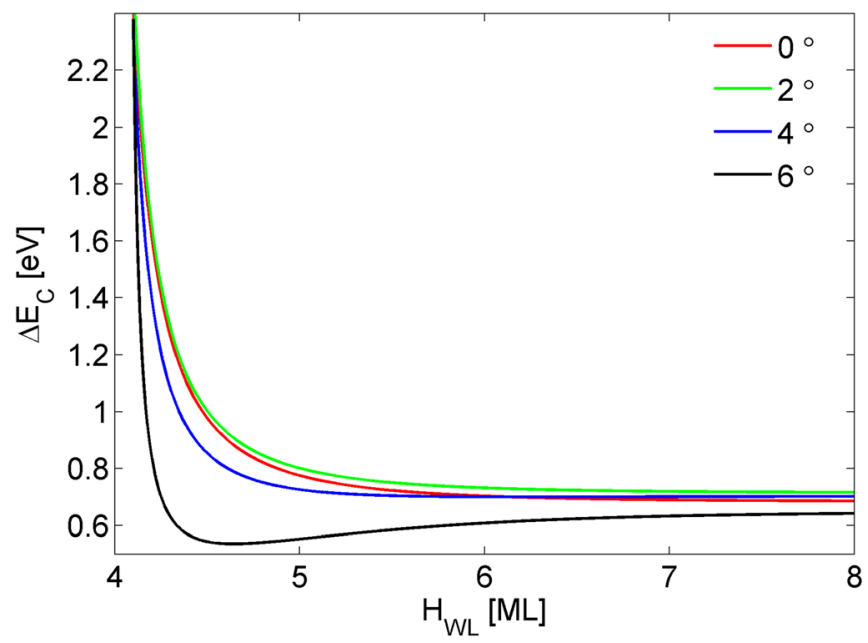

Finding the maximum of Eq. (6), the nucleation barrier and critical volume of island can be defined as

$$
\Delta E_{C}=\frac{4 Q^{3}}{27 P^{2}}, \quad V_{C}=\frac{8 Q^{3}}{27 P^{3}},
$$

where $P=a(1+\nu) W_{0} \tan \vartheta-K(\theta) \exp \left(-H_{W L}\right)\left(\gamma_{\text {sub }}^{\infty}-\gamma_{W L}^{\infty}\right) /$ $h_{0}$ and $Q=\gamma_{f} A_{2}(\theta)-K(\theta)\left[\gamma_{W L}^{\infty}-\left(\gamma_{W L}^{\infty}-\gamma_{s u b}^{\infty}\right) \exp \left(-H_{W L}\right)\right]$ $A_{1}(\theta)$.

\section{RESULTS AND DISCUSSION}

The thermodynamic model of nanoislands on vicinal substrate is established, and the nucleation barrier and critical volume is obtained by maximizing the formation energy. In general, the transition of growth mode has greatly influenced the physical properties of QDs, and the critical thickness of wetting layer which depends on the substrate misorientation is one of main parameters for determining the transition from 2D to 3D. To reveal the influence of vicinal substrate compared to the flat one, we take Ge/Si system and calculate the dependence of the formation nucleation barrier on the thickness of wetting layer with various miscut angles according to Eq. (7). In our calculation, we adopt $\gamma_{W L}^{\infty}=6.05 \mathrm{eV} / \mathrm{nm}^{2}, \gamma_{\text {sub }}^{\infty}=8.71 \mathrm{eV} / \mathrm{nm}^{2}{ }^{13}$ Other parameters are as follows: the Young's modulus $E$ of $103 \mathrm{GPa}$, the Poisson ratio $v$ of 0.26 , and the misfit parameter $\varepsilon_{0}$ of $4.2 \%$. The calculated results of nucleation barrier as a function of wetting layer thickness under different miscut angles are shown in Fig. 4(a). We notice that the nucleation barrier decreases with the increase of the thickness of wetting layer. Asymptotic values of the barrier for various miscut angles are almost the same. This is an expected result, because the barrier difference is not obvious in experimental observation, and this conclusion is also validated by the theoretical analysis. ${ }^{14}$ It is interesting and important to note that the minimum nucleation barrier of QDs corresponding to the wetting layer thickness is smaller for the large miscut substrate. Because of the low formation nucleation barrier, the QDs nucleation on large miscut substrate is favourable; hence, they are more preferred to take place with small thickness of wetting layer.

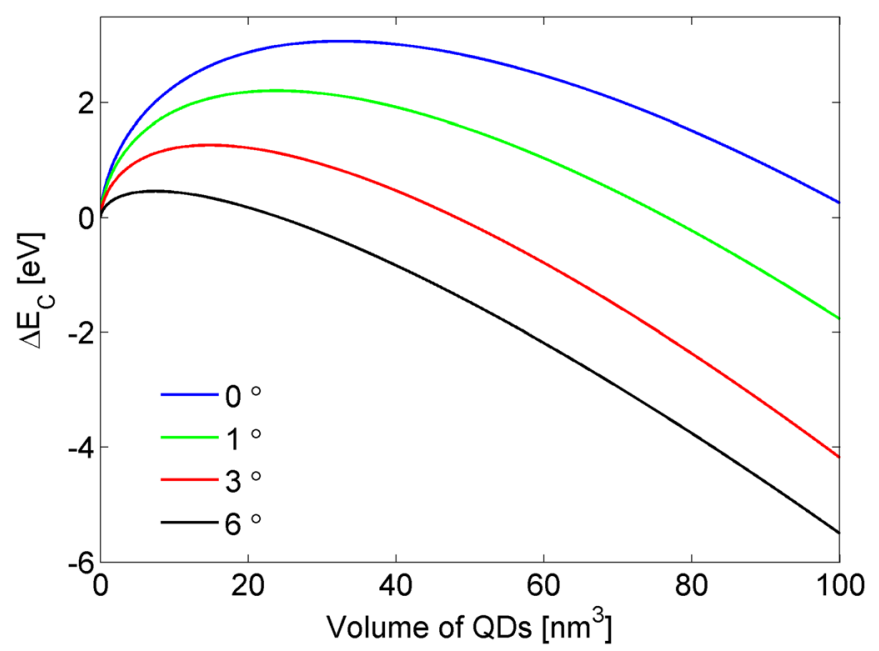

FIG. 4. (a) The nucleation barrier of QDs as a function of wetting layer thickness on $0^{\circ}, 2^{\circ}, 4^{\circ}, 6^{\circ}$ miscuts substrate, respectively. (b) The formation energy of QDs on $0^{\circ}, 1^{\circ}, 3^{\circ}, 6^{\circ}$ miscuts substrate, respectively, with fixed wetting layer thickness $H_{W L}=4.5 \mathrm{ML}$ and fixed density of QDs $n=100 \mu \mathrm{m}^{-2}$. 

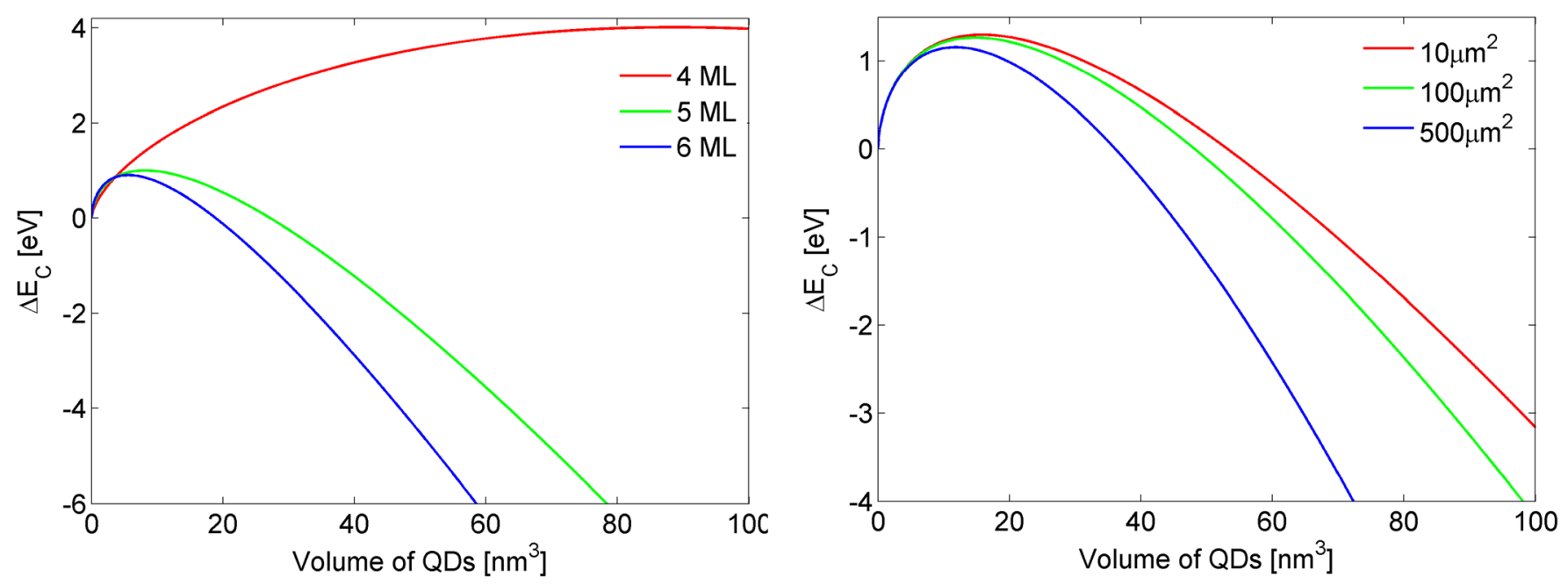

FIG. 5. The formation energy of QDs on $3^{\circ}$ miscuts substrate with (a) various wetting layer thicknesses, and the density of QDs equals $100 \mu \mathrm{m}^{-2}$. (b) various densities, and the thickness of wetting layer equals 4.5 monolayers.

Fig. 4(b) shows the results of the formation energy of QDs with fixed monolayer thickness and QD density under the conditions of $H_{W L}=4.5 \mathrm{ML}, n=100 \mu \mathrm{m}^{-2}$ and miscut angles $\theta=0^{\circ}, 1^{\circ}, 3^{\circ}, 6^{\circ}$, respectively. Clearly, we can see that the total energy rises with increasing volume of QDs initially, and then the total energy starts to decrease when the volume reaches the critical volume. The formation energy in correspondence with the critical volume is nucleation barrier. It is shown that the critical volumes of QDs grow smaller with the increase of miscut angle. This figure indicates that the nucleation barrier of islands on the vicinal substrate is lower than that of the flat one, and will become lower as the miscut angle increases. The experimental results have demonstrated that the formation of $\mathrm{Ge}\{105\}$ facets on the flat $\mathrm{Si}(001)$ surface is accompanied by an effective strain relaxation that overcompensates the increase in surface energy. ${ }^{15-17}$ Therefore, in the case of zero substrate miscut, the nucleation barrier arises because island formation raises the total surface energy. But at finite miscut, the surface energy of island is less than or equal to that of the vicinal wetting layer it covers, so there is less surface energy cost and the nucleation barrier decreases.

During the deposition process, the QDs density can be considered as a constant which is determined by the growth kinetics in the early nucleation phase. ${ }^{13}$ So, we calculate the formation energy as a function of QD volume under different wetting layer thicknesses and a fixed density as shown in Fig. 5(a). On the whole, both critical volume and nucleation barrier decrease with the increasing $H_{W L}$, which means it is more favorable for QDs to form with thicker WL. It is because the decreasing rate of surface energy of the WL with the increasing $H_{W L}$ becomes very small when the WL has a large thickness, so that the resistance of QD formation plays an unimportance role for a large $H_{W L}$. It is worthy of noting that QDs hardly form on the WL with the thickness of less than $4 \mathrm{ML}$. The conclusion is in good agreement with experimental result in which there is no QD formation on WL if the deposition amount is lower than 4 ML. ${ }^{18,19} \mathrm{In}$ Fig. 5(b), we can see that there are nearly the same critical volumes and nucleation barrier for the different densities. That is because the volume of QDs is small and the relative distance between QDs is large. Therefore, the QD density does not play an important role during the nucleation process.

The theoretical results demonstrate that when the volume of QDs larger than the critical volume, the growth mode of the QDs have a lower energy and is more favorable than that of the layer-by-layer mode, and the QDs can grow steadily due to the total energy decreasing with increasing volume. The competition between the surface energy and the elastic energy should be taken into account in nucleation process. The mismatch between the wetting layer and the substrate generate stress that induces instability. With the deposition thickness exceeds the critical value, the layer-bylayer can never proceed, in order to release the strain, the QDs nucleation takes place. There exists a strong influence of wetting layer on the QDs formation when the wetting layer thickness is small, which has been well proved by Muller and Thomas ${ }^{20}$

\section{CONCLUSIONS}

In summary, we have proposed a thermodynamic model to analyze the formation and the coherent growth mechanism of QDs on the strained vicinal substrate in which the density, the thickness-dependant wetting layer energy, and the miscut angle of the substrate are taken into account. A qualitative analysis based on the proposed new model is conducted on the critical formation energy and volume and the nucleation barrier of QDs on different parameters is also investigated. The theoretical predictions made in this paper are consistent with experimental results. Hence, the established approach in this paper provides a good way to understand selfassembled QDs on the vicinal substrate. The growth of QDs on strained vicinal substrate provides a convenient and effective method to control the size distribution of QDs. We expect new experimental design to obtain QDs with desired size and high quality. 


\section{ACKNOWLEDGMENTS}

This work was supported by the foundation research project of Henan Province under Contract No. 132300410072 and National Natural Science Foundation of China (No. 11104251).

${ }^{1}$ V. A. Shchukin and D. Bimberg, Rev. Mod. Phys. 71, 1125 (1999).

${ }^{2}$ D. Bimberg, M. Grundmann, and N. N. Ledentsov, Quantum Dot Heterostructures (Wiley, New York, 1999).

${ }^{3}$ V. G. Dubrovskii and X. Zhang, Tech. Phys. Lett. 37, 797 (2011).

${ }^{4}$ V. G. Dubrovskii, N. V. Sibirev, X. Zhang, and R. A. Suris, Cryst. Growth Des. 10, 3949 (2010).

${ }^{5}$ V. G. Dubrovskii, G. E. Cirlin, and V. M. Ustinov, Phys. Rev. B 68, 075409 (2003).

${ }^{6}$ A. V. Osipov, S. A. Kukushkin, F. Schmitt, and P. Hess, Phys. Rev. B 64, 205421 (2001).

${ }^{7}$ L. Persichetti, A. Sgarlata, M. Fanfoni, and A. Balzarotti, Phys. Rev. Lett. 104, 036104 (2010).

${ }^{8}$ L. Persichetti, A. Sgarlata, M. Fanfoni, and A. Balzarotti, Phys. Rev. B 82, 121309(R) (2010).
${ }^{9}$ I. V. Markov, Crystal Growth for Beginners: Fundamentals of Nucleation, Crystal Growth, and Epitaxy (World Scientific, Singapore, 1995).

${ }^{10}$ P. D. Szkutnik, A. Sgarlata, A. Balzarotti, N. Motta, A. Ronda, and I. Berbezier, Phys. Rev. B 75, 033305 (2007).

${ }^{11}$ V. A. Shchukin, D. Bimberg, T. P. Munt, and D. E. Jesson, Phys. Rev. B 70, 085416 (2004)

${ }^{12}$ B. J. Spencer and J. Tersoff, Appl. Phys. Lett. 96, 073114 (2010).

${ }^{13}$ X. L. Li and G. W. Yang, Appl. Phys. Lett. 92, 171902 (2008).

${ }^{14}$ Xu. Zhang, N. V. Sibirev, V. G. Dubrovskii, and X. Ren, 19th International Symposium on Nanostructures: Physics and Technology, 2011.

${ }^{15}$ V. B. Shenoy, C. V. Ciobanu, and L. B. Freund, Appl. Phys. Lett. 81, 364 (2002).

${ }^{16}$ G. H. Lu, M. Cuma, and F. Liu, Phys. Rev. B 72, 125415 (2005).

${ }^{17}$ G. H. Lu and F. Liu, Phys. Rev. Lett. 94, 176103 (2005).

${ }^{18}$ D. N. Lobanov, A. V. Novikov, N. V. Vostokov, Y. N. Drozdov, A. N. Yablonskiy, Z. F. Krasilnik, M. Stoffel, U. Denker, and O. G. Schmidt, Opt. Mater. 27, 818 (2005).

${ }^{19}$ D. V. Yurasov, Yu. N. Drozdov, M. V. Shaleev, and A. V. Novikov, Appl. Phys. Lett. 95, 151902 (2009).

${ }^{20}$ P. Muller and O. Thomas, Surf. Sci. 465, L764 (2000). 